\title{
Improved Meet-in-the-Middle Attacks on Reduced-Round DES ${ }^{\star}$
}

\author{
Orr Dunkelman, Gautham Sekar, and Bart Preneel \\ Katholieke Universiteit Leuven \\ Department of Electrical Engineering ESAT/SCD-COSIC \\ Kasteelpark Arenberg 10, B-3001 Leuven-Heverlee, Belgium \\ \{orr. dunkelman, gautham. sekar, bart.preneel\}@esat. kuleuven. be
}

\begin{abstract}
The Data Encryption Standard (DES) is a 64-bit block cipher. Despite its short key size of 56 bits, DES continues to be used to protect financial transactions valued at billions of Euros. In this paper, we investigate the strength of DES against attacks that use a limited number of plaintexts and ciphertexts. By mounting meet-in-the-middle attacks on reduced-round DES, we find that up to 6-round DES is susceptible to this kind of attacks. The results of this paper lead to a better understanding on the way DES can be used.
\end{abstract}

\section{Introduction}

The Data Encryption Standard (DES) is a well known and widely deployed cipher since its standardization in 1977. Its wide deployment, even today, makes it a target for repeated analyses, as the security of many electronic transactions still relies on DES. The cipher is a Feistel block cipher with 16 rounds, 64-bit block and 56-bit key.

Due to its importance, DES has received a great deal of cryptanalytic attention. However, besides using the complementation property, there were no short-cut attacks against the cipher until differential cryptanalysis was applied to the full DES in 1991 [2-4].

In [5], Chaum and Evertse presented several meet-in-the-middle attacks on reduced variants of DES. They showed that the first six round of DES are susceptible to meet-in-the-middle attacks, such as rounds $2-8$. They also showed that their approach cannot be extended to more than seven rounds of DES.

In 1987 Davies described a known plaintext attack on DES [8]. The attack obtains 16 linear equations of the key bits given sufficiently many known plaintexts by examining the bits that are shared by neighboring S-boxes. Davies' on the full DES requires more plaintexts than the entire code book. For 8-round

\footnotetext{
* This work was supported in part by the Concerted Research Action (GOA) Ambiorics 2005/11 of the Flemish Government and by the IAP Programme P6/26 BCRYPT of the Belgian State (Belgian Science Policy). The second author is supported by an IWT SoBeNeT project. This is a revised version of the paper published in the proceedings of Indocrypt 2007.
} 
DES, the attack requires about $2^{40}$ known plaintexts. In [9] these results were slightly improved but still could not attack the full DES faster than exhaustive key search. In 1994 Biham and Biryukov [1] improved the attack to be applicable to the full DES. Their variant of the attack requires $2^{50}$ known plaintexts and has a running time of $2^{49}$ encryptions on an average. A chosen ciphertext variant of the attack is presented in [15]; it has a data complexity of $2^{45}$ chosen plaintexts.

The first attack on DES that is faster than exhaustive key search was presented in [4]. The attack, differential cryptanalysis, requires $2^{47}$ chosen plaintexts. The attack examines pairs of plaintexts and ciphertexts, trying to find a pair that satisfies some differential (i.e., given some input difference between the two plaintexts, the output difference of the two ciphertexts is as predicted). Once such a pair is found, the key can be deduced.

In [16] another attack on DES is presented, linear cryptanalysis. The linear attack on DES uses $2^{43}$ known plaintexts and deduces the key by checking whether some linear relation between the plaintext and the ciphertext is satisfied. This attack was later improved in [21] by exploiting nonlinear relations as well. The improved attack has a data complexity of $2^{42.6}$ known plaintexts. Using chosen plaintexts, Knudsen and Mathiassen reduced the data complexity in [16] by a factor of 2 .

Even after DES was theoretically broken, it was claimed that DES was still secure, as it was not possible to mount these attacks in practice. As a response, RSA Data Security Inc. has issued several "DES Challenges" during the mid '90s. In each such challenge, RSA published a plaintext and its ciphertext encrypted using DES under some unknown key, and offered a prize of several thousand US dollars for whoever finds the secret key [6]. The first exhaustive key search took about 75 days and the key was found using 14,000-80,000 computers over the Internet [22]. Ever since, the time required for each new DES challenge has been reduced. In 1997 the Electronic Frontier Foundation (EFF) built a special purpose machine that costs 250,000 US dollars which retrieved the key in 56 hours by means of exhaustive key search [11]. Today, using a COPACOBANA machine an exhaustive key search of DES can be performed in 17 days for the cost of less than 9000 Euros [14].

Recently, a new approach was presented by Raddum and Semaev for solving sparse systems of non-linear equations and used them to attack up to 4 rounds of DES [19]. With 16 known plaintext-ciphertext pairs, their techniques produce an equation system with 1080 variables and 2048 non-linear equations from 4round DES. While their methods work on 5 or more rounds of DES, they are too complex to be considered in practice.

The approach of treating reduced-round DES as an algebraic equation was also suggested in [7]. The attack described here represents DES as a system of multivariate equations with the key bits as unknowns and tries to solve the system using SAT solvers. This technique can find the key with up to six rounds of DES faster than exhaustive key search. 
Motivation behind our work: Despite the well known weaknesses of DES, the cipher is still widely deployed and used. In addition, DES-like ciphers are being suggested as a solution for encryption in RFID systems [18].

All the existing attacks on DES either use a long time (exhaustive key search) or use a very large number of plaintexts. This motivated us to investigate how many rounds of DES can be broken using the meet-in-the-middle technique, using one (or very few) plaintexts. We aimed at finding the best attacks on reduced-round DES. The results of this paper shed more light on the security of DES, leading to a better understanding on the way DES can be used.

Contribution of this paper: We have devised a new approach to meetin-the-middle attacks. Rather than guessing all the key bits that are required to produce some value, our approach guesses actual intermediate encryption values, thus saving the need to guess many key bits to obtain the value of an intermediate encryption bit. This improves greatly on the previous attacks on reduced-round DES by Chaum and Evertse [5].

The new approach reduces the time complexity of the meet-in-the-middle attacks, as it allows for guessing significantly less number of key bits. Moreover, by obtaining several known plaintexts, one can increase the number of intermediate encryption bits that are guessed while decreasing the total time complexity of the attack. This follows from the fact that even if with only one of the known plaintexts, a specific key guess has no possible intermediate encryption value which fits the meet-in-the-middle condition, then the key guess is necessarily wrong.

Another possible use of our approach is in the chosen text scenario, where by fixing some bits of the plaintext (or the ciphertext), it is possible to force the intermediate values of several plaintext/ciphertext pairs to a specific value. This leads to a reduction in the number of bits that the attacker needs to guess (across several plaintext/ciphertext pairs).

This approach may also be used to improve other meet-in-the-middle attacks. To the best of our knowledge this is the first case where the attacker guesses intermediate encryption values rather than keys in a meet-in-the-middle attack. In this paper, we also provide insights into how our attacks might be extended to attack DES with more than 6 consecutive rounds using a similar approach as described above.

We compare the results of our attack with other attacks in in Table 1. We note that for differential and linear cryptanalysis we used a lower bound based on a linear attack with one active S-box in the round before and a round after the approximation is used. We used a similar lower bound for a differential attack on DES (taking into consideration a $3 \mathrm{R}$ attack). We also note that these attacks have two properties which make them inferior to our results: first of all, these attacks are statistical, i.e., while our approach ensures finding the key, statistical attacks may fail. In the table we mentioned the complexities of these attacks with at least $90 \%$ success rate. The second property is that the mentioned time complexities for these attacks is the time complexity required to retrieve several key bits, while our complexities are mentioned for finding the entire key. 


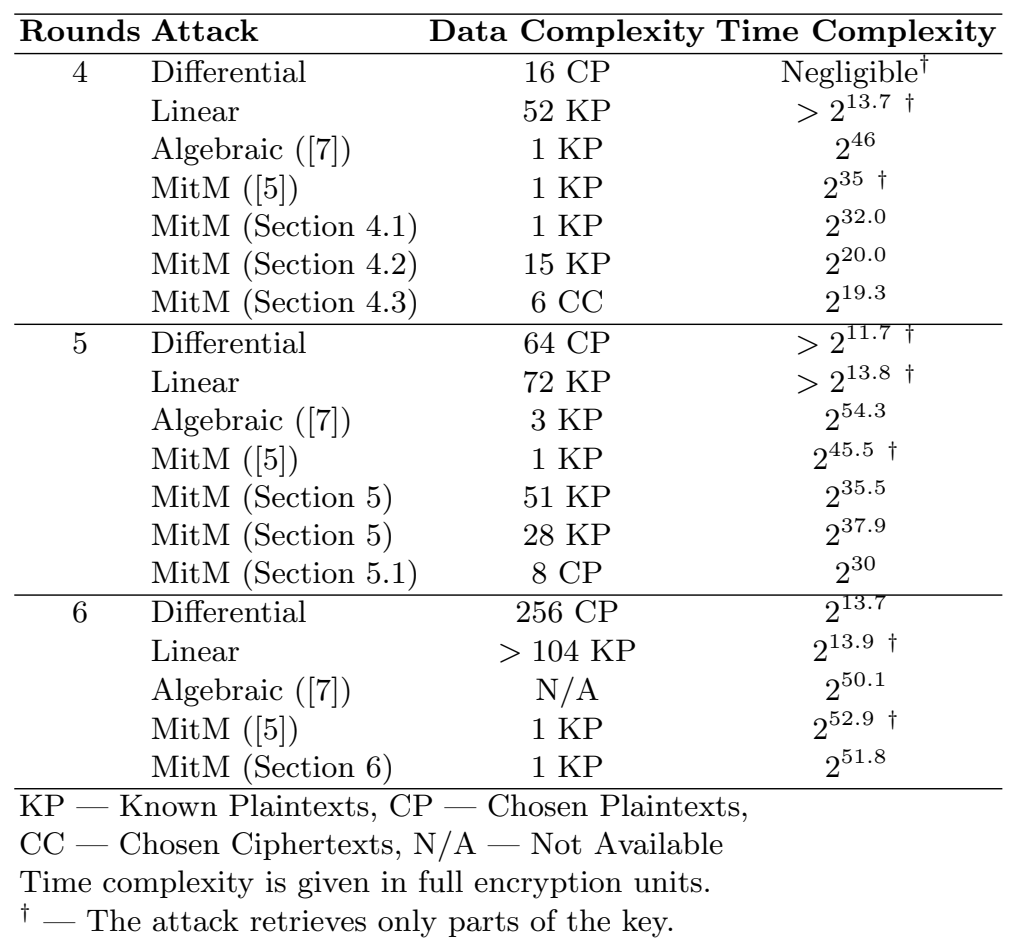

Table 1. Comparison of Attacks on Reduced-Round DES

The paper is organized as follows: Sect. 2 describes DES. In Sect. 3 we give an alternative description of DES and give the notations used in this paper. Our attack on 4-round DES is described in Sect. 4. Our results on 5-round and 6round DES are described in Sect. 5 and Sect. 6, respectively. Finally, we present our conclusions and a few open problems in Sect. 7 .

\section{Description of DES}

The Data Encryption Standard (DES) was accepted as the American standard in 1977 and became a de-facto standard for most protocols around the world [17]. DES is a 16-round Feistel block cipher, which accepts a 64-bit block and encrypts it under a 56-bit key. The input is divided into two halves, left and right, each consisting of 32 bits. The round function is applied 16 times to the two halves. In each round, the right half enters the $F$-function of DES along with the round's subkey. The output of $F$ is XORed to the left half. Then, the two halves are swapped. We give the outline of DES in Figure 1.

Let $I P(x)$ be the operation of permuting a vector $x \in\{0,1\}^{64}$ according to the initial permutation, and let $F P(x)$ be the final permutation. These permutations satisfy $F P=I P^{-1}$. As both $I P(\cdot)$ and $F P(\cdot)$ have no cryptographic 


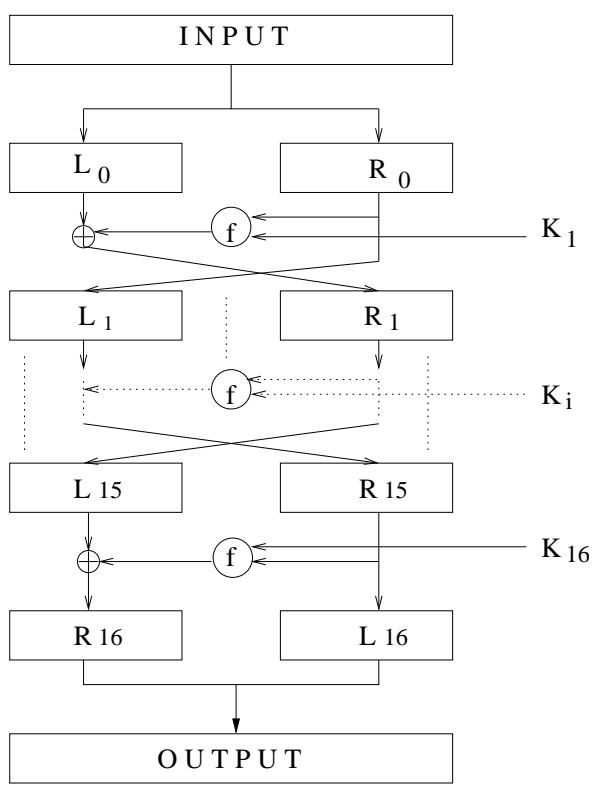

Fig. 1. An alternative description of the general structure of the Data Encryption Standard

effect, we disregard their existence. Let $L_{i n}, R_{i n}$ be the left and right halves, respectively, entering the round, and let $L_{\text {out }}, R_{\text {out }}$ be the left and right halves that the round outputs. Then, the round function is denoted by $\left(L_{\text {out }}, R_{\text {out }}\right)=$ $\operatorname{Round}_{K_{r}}\left(L_{i n}, R_{i n}\right)$ and $K_{r} \in\{0,1\}^{48}$ is the round subkey. Given this setting, one round of DES (without the swap of the Feistel construction) is represented by $R_{\text {out }}=R_{\text {in }}, L_{\text {out }}=L_{\text {in }} \oplus F\left(R_{\text {in }}, K_{r}\right)$.

The $F$-function of DES accepts an input of 32 bits along with a 48-bit subkey. The input is expanded into 48 bits (by duplicating 16 of the 32 input bits), and the expanded input is XORed with the subkey. The 48-bit outcome is divided into eight groups of six bits each. Each group enters a $6 \times 4$ S-box which is a nonlinear look up table. The same eight S-boxes $S 1, S 2, \ldots, S 8$ are applied in the same order in each round. The output of the S-boxes is permuted according to some permutation table $P$, and becomes the output of $F$. The outline of $F$ is given in Fig. 2.

The key schedule algorithm of DES takes as an input the 56-bit user supplied key, $K$, and produces 16 subkeys, $K_{1}, \ldots, K_{16}$, where each subkey is 48 bits long. The algorithm uses two tables namely, Permuted Choice-1 $(P C-1)$ and Permuted Choice-2 (PC-2). For most applications discussed in this paper, the details of how the subkeys are derived are not important, therefore, we omit its full description and refer the reader to [17]. 


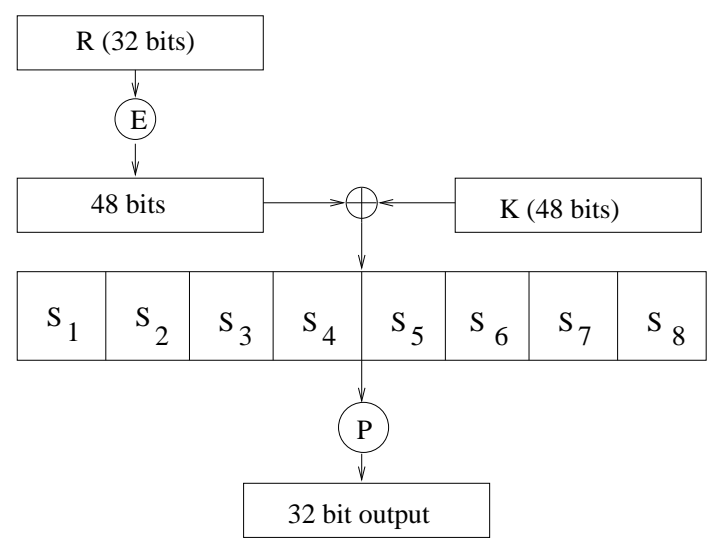

Fig. 2. $F$-function of DES

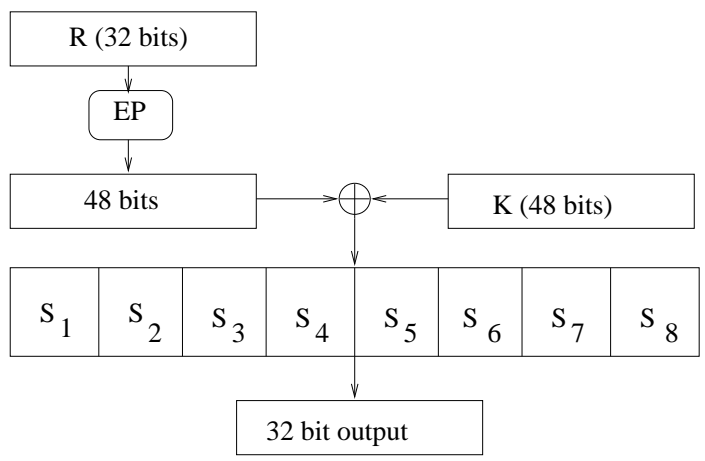

Fig. 3. An alternative description of DES' $F$-function

\section{An Alternative Description of DES and Notations Used}

Since this paper is based on [5], we retain the same alternative description of DES used by Chaum and Evertse. In their alternative description of DES, IP, FP, $P C-1$ are not used and $E, P$ are combined into one table $E P$. This model makes the description of the results more clear, while not affecting the correctness of the result. The $F$-function of the alternative description is illustrated in Fig. 3

Let $K$ denote the full 56-bit user supplied key. Following [17], we use the big endian notations, i.e., 'bit 1' is the most significant bit of the key, and 'bit 56 ' is the least significant bit of the key. We denote the $i$-th subkey by $K_{i}$. Finally, let $Y$ be some variable (an intermediate encryption value or a key). We use $Y$ [a-b] to denote bits $a, \ldots, b$ of $Y$. 


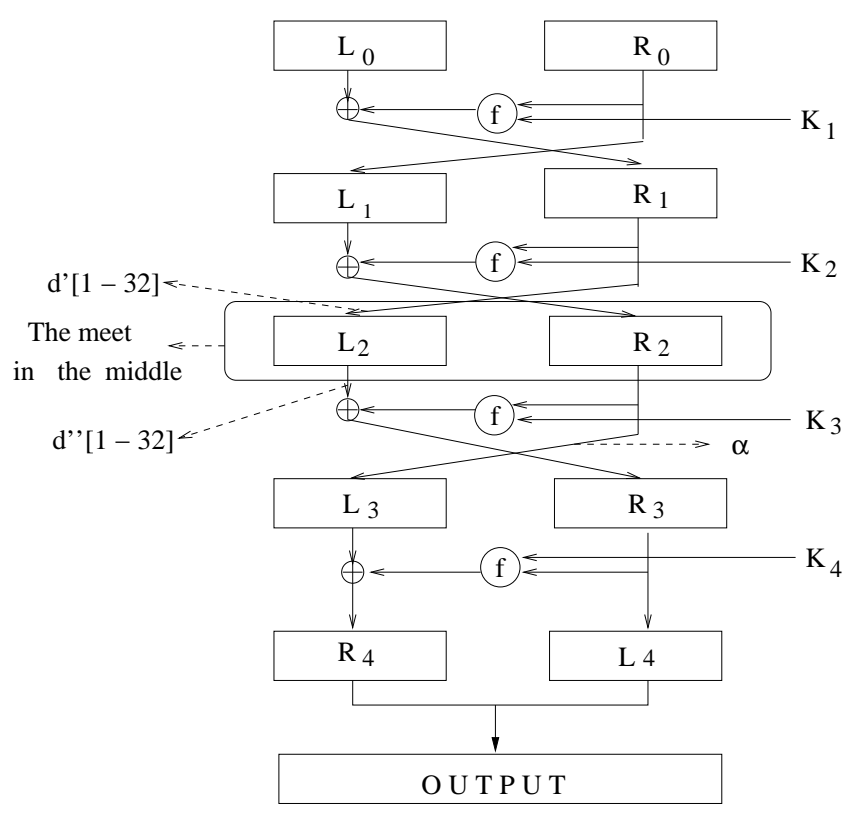

Fig. 4. 4-Round DES

\section{Meet-in-the-Middle Attack on 4-round DES}

We now describe our attack on 4-round DES. First, we start with a short description of meet-in-the-middle attacks. Let $\mathcal{M}$ denote the message space and $\mathcal{K}$ denote the key space. Suppose that $G_{K}, H_{K}: \mathcal{M} \times \mathcal{K} \rightarrow \mathcal{M}$ are two block ciphers and let $F_{K}=H_{K} \circ G_{K}$. In a meet-in-the-middle attack, the attacker tries to deduce $K$ from a given plaintext ciphertext pair $c=F_{K}(p)$ by trying to solve

$$
G_{K}(p)=H_{K}^{-1}(c) .
$$

In some of the cases, the equation is not tested for all the bits of the intermediate encryption value, but rather to only some of them.

Let $d^{\prime}[1-\mathrm{m}]=G_{K}(p)$ and $d^{\prime \prime}[1-\mathrm{m}]=H_{K}^{-1}(c)$. In our attack on 4-round DES, as in attack by Chaum and Evertse, $G_{K}$ consists of the first 2 rounds of DES and $H_{K}$ contains of rounds 3 and 4 . Let us consider $d^{\prime}[9-12]$ and $d^{\prime \prime}[9-12]$ as illustrated in Fig. 4.

It was observed in [5] that in order to compute $d^{\prime}[9-12]$ and $d^{\prime \prime}[9-12]$, it is sufficient to guess only 37 key bits. Thus, if for a key guess the computed values of $d^{\prime}[9-12]$ and $d^{\prime \prime}[9-12]$ disagree, then the key guess cannot be correct (as it leads to contradiction) and can be discarded.

Our main observation is the fact that the values of $d^{\prime}[9-12]$ and $d^{\prime \prime}[9-12]$ can be computed by guessing less key bits in exchange for guessing internal bits. 
Consider $d^{\prime}[9-12]$, this value is equal to:

$$
d^{\prime}[9-12]=L_{0}[9-12] \oplus S_{3}\left[E P\left(R_{0}\right)[13-18] \oplus K_{1}[13-18]\right]
$$

and $d^{\prime \prime}[9-12]$ is equal to

$$
d^{\prime \prime}[9-12]=L_{4}[9-12] \oplus S_{3}\left[E P\left(L_{3}\right)[13-18] \oplus K_{3}[13-18]\right] .
$$

Let $L_{3}=\left[\alpha_{1}-\alpha_{32}\right]$, then

$$
E P\left(L_{3}\right)[13-18]=\left[\alpha_{17} \alpha_{1} \alpha_{15} \alpha_{23} \alpha_{26} \alpha_{5}\right] .
$$

If we guess $K_{1}[13-18]$ and $K_{3}[13-18]$, the only remaining unknowns in the computation of $d^{\prime \prime}[9-12]$ are $\left[\alpha_{17} \alpha_{1} \alpha_{15} \alpha_{23} \alpha_{26} \alpha_{5}\right]$.

Consider $\alpha_{17}$. In order to compute this bit we can either guess key bits $K_{4}[25-30]$ or guess $\alpha_{17}$ directly. Thus, a different attack algorithm for the meetin-the-middle attack would be to guess all the 37 key bits suggested by Chaum and Evertse, besides the 6 bits which compose $K_{4}$ [25-30]. For each guess of the 31 key bits, the attacker tries the two possibilities of $\alpha_{17}$. If for both values the equality $d^{\prime}[9-12]=d^{\prime \prime}[9-12]$ is not achieved, then the guess of the 31 bits is necessarily wrong. As for a specific (wrong) guess of the key and of $\alpha_{17}$ the probability of equality is $1 / 16$, the probability that a wrong 31-bit key guess has at least one $\alpha_{17}$ for which the equality is satisfied is $1-(15 / 16)^{2} \approx 1 / 8$. Hence, the attacker can guess 31 bits, and by trying the two possibilities of $\alpha_{17}$ reduce the number of remaining candidates to $2^{28}$. From this point, the attacker can either repeat Chaum and Evertse's original attack or use a more advanced approach. In Table 3 we list the required key bits for determining $d^{\prime}[9-12]$ and $d^{\prime \prime}[9-12]$ and note which of the key bits are used only once in the computations of $d^{\prime}$ and $d^{\prime \prime}$.

An attacker can guess several $\alpha_{i}$ values simultaneously, thus reducing the number of possible keys (in exchange for increasing the probability that a wrong key remains). For example, if the attacker guesses two intermediate encryption bits, the probability that a key remains is $1-(15 / 16)^{4} \approx 2^{-2.1}$. For three and four intermediate bits the remaining probabilities are $2^{-1.3}$ and $2^{-0.6}$, respectively. This approach can lead, in the extreme, to the following meet-in-the-middle attack:

\subsection{A Meet-in-the-Middle Attack with One Known Plaintext}

We first define a procedure to analyze a meet-in-the-middle attack on a specific S-box. Attacking $S x$ in round 2 means that we guess the key which enters this S-box, as well as $S x$ in round 4 (in order to determine their outputs). We also need to know the 6 bits which enter this S-box, i.e., we need to know the output of 6 S-boxes in round 1. For example, performing a meet-in-the-middle on $S 3$ of round 3 involves guessing $K_{1}[1-12], K_{1}[19-24], K_{2}[13-18], K_{4}[13-18]$ (a total of 19 bits), and guessing 3 intermediate encryption values $\left(\delta_{17}, \delta_{23}, \delta_{26}\right.$; where $\left.L_{2}=\left[\delta_{1}-\delta_{32}\right]\right)$. Thus, it is expected that after such an analysis, of the $2^{19}$ possible 


\begin{tabular}{|c|c|c|c|c|}
\hline \multirow[t]{2}{*}{ Round } & \multirow[t]{2}{*}{ S-box } & \multicolumn{2}{|c|}{ Number of Guessed } & \multirow{2}{*}{$\begin{array}{c}\text { Number of Remaining } \\
\text { Key Guess }\end{array}$} \\
\hline & & Key Bits & Intermediate Bits & \\
\hline 3 & $S 3$ & 19 & 3 & $2^{19} \cdot 2^{-1.3}=2^{17.7}$ \\
\hline 3 & $S 2$ & +3 & 4 & $2^{17.7} \cdot 2^{3} \cdot 2^{-0.6}=2^{20.1}$ \\
\hline 2 & $S 1$ & +2 & 4 & $2^{20.1} \cdot 2^{2} \cdot 2^{-0.6}=2^{21.5}$ \\
\hline 3 & $S 4$ & +3 & 3 & $2^{21.5} \cdot 2^{3} \cdot 2^{-1.3}=2^{23.2}$ \\
\hline $2^{\dagger}$ & $S 4$ & +1 & 3 & $2^{23.2} \cdot 2^{1} \cdot 2^{-1.3}=2^{22.9}$ \\
\hline 3 & $S 3$ & - & 3 & $2^{22.9} \cdot 2^{-1.3}=2^{21.6}$ \\
\hline 2 & $S 2$ & - & 4 & $2^{21.6} \cdot 2^{-0.6}=2^{21.0}$ \\
\hline 3 & $S 1$ & - & 4 & $2^{21.0} \cdot 2^{-0.6}=2^{20.4}$ \\
\hline 2 & $S 8$ & +9 & $2(-2)^{\ddagger}$ & $2^{20.4} \cdot 2^{9} \cdot 2^{-4}=2^{25.4}$ \\
\hline 3 & $S 5$ & +5 & $1(-5)^{\ddagger}$ & $2^{25.4} \cdot 2^{5} \cdot 2^{-8}=2^{22.4}$ \\
\hline 3 & $S 6$ & +4 & $2(-5)^{\ddagger}$ & $2^{22.4} \cdot 2^{4} \cdot 2^{-7}=2^{19.4}$ \\
\hline 2 & $S 7$ & +4 & $1(-4)^{\ddagger}$ & $2^{19.4} \cdot 2^{4} \cdot 2^{-7}=2^{16.4}$ \\
\hline 3 & $S 7$ & +3 & $2(-5)^{\ddagger}$ & $2^{16.4} \cdot 2^{3} \cdot 2^{-7}=2^{12.4}$ \\
\hline 3 & $S 8$ & +2 & $1(-9)^{\ddagger}$ & $2^{12.4} \cdot 2^{2} \cdot 2^{-12}=2^{2.4}$ \\
\hline \multicolumn{5}{|c|}{ Exhaustively search the remaining $2^{3.4}$ keys. } \\
\hline \multicolumn{5}{|c|}{$\begin{array}{l}\text { - At this point the entire half of the key is known. } \\
\text { - The }(-i) \text { means that there } i \text { bits that were earlier guessed and are } \\
\text { now known (and can be used to discard wrong inconsistent guesses). }\end{array}$} \\
\hline
\end{tabular}

Table 2. Meet-in-the-middle on 4-round DES with one known plaintext

values for the 19-bit key, only $2^{17.7}$ values remain. Similarly, one can define a meet-in-the-middle attack on $S x$ in round 3 (while guessing the key of $S x$ in round 1 , and the output of 6 S-boxes in round 4).

To describe the attack algorithm, we give the sequence of attacked S-boxes. For each step, we give the number of additional key bits to be guessed, along with the number of intermediate bits that the attacker has to guess, and the number of remaining key guesses after the S-box is attacked. The attacker can retrieve the full key using about $2^{32.0} 4$-round DES encryptions by attacking the sequence of S-boxes given in Table 2.

\subsection{Using Multiple Known Plaintexts}

If several plaintext/ciphertext pairs are at the disposal of the attacker, they can be used to deduce the value of the first 19 guessed bits in a more efficient way. The attacker uses the first plaintext/ciphertext pair to reduce the number of possible keys to $2^{17.7}$. Then, using the next plaintext/ciphertext pair, he repeats the analysis (with less candidates for the 19 bits of the key). As the probability that a key remains after each iteration of the analysis is $1-(15 / 16)^{8} \approx 0.4$, the number of trials $t$ required for discarding all the wrong keys satisfies: $2^{19} \cdot 0.4^{t}<1$. Thus, after 15 plaintext/ciphertext pairs, we expect to have only the right value for 19 key bits, which can then be used to retrieve the remaining key bits in a similar manner. 


\begin{tabular}{|c|c|c|c|}
\hline Round/S-box & Key bits & Bit determined & Bits appearing once ${ }^{\dagger}$ \\
\hline $1 / 3$ & $5,9,13,20,24,27$ & & 24 \\
$3 / 3$ & $2,8,12,16,23,27$ & & $7,11,17$ \\
$4 / 1$ & $2,7,11,17,20,23$ & $\alpha_{1}$ & 6,21 \\
$4 / 2$ & $6,9,12,16,21,27$ & $\alpha_{5}$ & $19,22,26$ \\
$4 / 4$ & $5,8,13,19,22,26$ & $\alpha_{15}$ & $30,33,37,43,47,53$ \\
$4 / 5$ & $30,33,37,43,47,53$ & $\alpha_{17}$ & $29,36,39,46,51,54$ \\
$4 / 6$ & $29,36,39,46,51,54$ & $\alpha_{23}$ & $31,34,40,45,50,55$ \\
$4 / 7$ & $31,34,40,45,50,55$ & $\alpha_{26}$ \\
\hline Bits of $K$ & \multicolumn{3}{|c}{$1,3,4,10,14,15,18,25,28,32,35,38,41,42,44,48,49,52,56$} \\
not affecting $(1)$ & \multicolumn{2}{|c}{} \\
\hline
\end{tabular}

${ }^{\dagger}$ — These bits appear only once in computing $d^{\prime}$ and $d^{\prime \prime}$.

Table 3. Key bits determining the 'middle' bits of 4-round DES

The time complexity of the attack in this case is about $2^{20}$ full 4 -round DES encryptions (there are $2^{19}$ keys, and $2^{3}$ intermediate values to check for each of them).

\subsection{Using Chosen Ciphertexts}

It is also possible to use chosen ciphertexts to improve the data complexity of the known plaintext attack. If we choose the ciphertexts in such a way that the intermediate encryption bits which are guessed are the same for all the ciphertexts, we actually improve the filtering each new plaintext/ciphertext pair offers. This follows the fact that in the known plaintext scenario, each plaintext/ciphertext pair may "allow" a key guess to pass due to a different value in the intermediate encryption values. In the chosen ciphertext scenario, the attacker guesses the 19 key bits. A key which is not discarded, but has less than 8 possible intermediate encryption values (which is the case for most of the keys), is tested with the next plaintext/ciphertext pair only with the intermediate encryption values which satisfied the meet-in-the-middle condition earlier.

Thus, a given key has probability 0.6 to be discarded with the first plaintext/ciphertext pair, probability 0.32 to pass to the next pair with only one candidate value for the intermediate encryption bits, probability 0.074 to pass to the next pair with two possible values in the intermediate encryption values, and so forth. Thus, it is expected that the next pair discards 15 out of 16 remaining keys with one value, and about 14 out of 16 keys remaining with one value (while reducing the number of possible intermediate encryption values of most of them to 1 ). We conclude that 6 chosen ciphertexts are sufficient to find the first 19 key bits (from where by repeating the previous attacks we can find the rest of the key). The running time of the attack is $2^{19.3}$ encryptions. 


\section{$5 \quad$ Attack on 5-round DES}

The attack on 5-round DES proceeds along the same lines as the attack on 4-round DES. Let $d^{\prime}[1-\mathrm{m}]=G_{K}(p)$ and $d^{\prime \prime}[1-\mathrm{m}]=H_{K}^{-1}(c)$. In our attack on 5round DES, as in attack by Chaum and Evertse, $G_{K}$ consists of the first 2 rounds of DES and $H_{K}$ contains of rounds 3,4 and 5. Let us consider the intermediary bits $d^{\prime}[41-44]$ and $d^{\prime \prime}[41-44]$.

We present the results of our analysis of 5 -round DES in Table 4. It was observed in [5] that in order to compute $d^{\prime}[41-44]$ and $d^{\prime \prime}[41-44]$, it is sufficient to guess only 47 key bits. Thus, if for a key guess the computed values of $d^{\prime}[41-44]$ and $d^{\prime \prime}[41-44]$ disagree, then the key is necessarily wrong, and can be discarded.

Again, the values of $d^{\prime}[41-44]$ and $d^{\prime \prime}[41-44]$ can be computed by guessing less key bits in exchange for guessing the values of intermediate bits. Consider $d^{\prime}[41-44]$, this value is equal to:

$$
d^{\prime}[41-44]=R_{0}[9-12] \oplus S_{3}\left[E P\left(R_{1}\right)[13-18] \oplus K_{2}[13-18]\right]
$$

and $d^{\prime \prime}[41-44]$ is equal to

$$
d^{\prime \prime}[41-44]=L_{5}[9-12] \oplus S_{3}\left[E P\left(L_{4}\right)[13-18] \oplus K_{4}[13-18]\right] .
$$

Let $R_{1}=\left[\beta_{1}-\beta_{32}\right], L_{4}=\left[\gamma_{1}-\gamma_{32}\right]$. Then,

$$
\begin{aligned}
& E P\left(R_{1}\right)[13-18]=\left[\beta_{17} \beta_{1} \beta_{15} \beta_{23} \beta_{26} \beta_{5}\right], \\
& E P\left(L_{4}\right)[13-18]=\left[\gamma_{17} \gamma_{1} \gamma_{15} \gamma_{23} \gamma_{26} \gamma_{5}\right] .
\end{aligned}
$$

If we guess $K_{2}[13-18]$ and $K_{4}[13-18]$, the only unknowns left in the computations of $d^{\prime}[41-44]$ and $d^{\prime \prime}[41-44]$ are $\left[\beta_{17} \beta_{1} \beta_{15} \beta_{23} \beta_{26} \beta_{5}\right]$ and $\left[\gamma_{17} \gamma_{1} \gamma_{15} \gamma_{23} \gamma_{26} \gamma_{5}\right]$.

Consider $\beta_{1}$. In order to compute this bit we can either guess 2 key bits (of $K_{1}[1-6]$ ) or guess $\beta_{1}$ directly. Thus, a different attack algorithm for the meet-in-the-middle attack would be to guess all the 47 key bits suggested by Chaum and Evertse, besides the 2 key bits. For each guess of the 45 key bits, the attacker tries the two possibilities of $\beta_{1}$. If for both values the equality $d^{\prime}[41-44]=d^{\prime \prime}[41-44]$ is not achieved, then the guess of the 45 bits is necessarily wrong. As for a specific (wrong) guess of the key and of $\beta_{1}$ the probability of equality is $1 / 16$, the probability that a wrong 45 -bit key has at least one $\beta_{1}$ for which the equality is satisfied is $1-(15 / 16)^{2} \approx 1 / 8$. Hence, the attacker can guess the $45 \mathrm{bits}$, and by trying the two possibilities of $\beta_{1}$ reduce the number of remaining candidates to $2^{42}$. From this point, the attacker can either repeat Chaum and Evertse's original attack or use a more advanced approach along similar lines as the method described in Sect. 4.2.

However, a more efficient attack exists. We note that there are many key bits which are used twice in determining the values of $\beta_{17}, \beta_{23}, \beta_{26}$ and $\gamma_{17}, \gamma_{23}$, and $\gamma_{26}$. Thus, it is still more efficient to guess the values of $\beta_{23}, \beta_{26}, \gamma_{17}$, and $\gamma_{26}$ than guessing these key bits directly. More precisely, to determine $\beta_{17}$ and $\gamma_{23}$ 


\begin{tabular}{|c|c|c|c|}
\hline Round/S-box & Key bits & Bit determined & Bits appearing once \\
\hline $1 / 1$ & $2,6,12,15,18,25$ & $\beta_{1}$ & 2,12 \\
$1 / 2$ & $1,4,7,11,16,22$ & $\beta_{5}$ & 16 \\
$1 / 4$ & $3,8,14,17,21,28$ & $\beta_{15}$ & 3,17 \\
$1 / 5$ & $32,38,42,48,53,56$ & $\beta_{17}$ & 34,46 \\
$1 / 6$ & $31,34,41,46,49,52$ & $\beta_{23}$ & $40,50,54$ \\
$1 / 7$ & $29,35,40,45,50,54$ & $\beta_{26}$ & \\
$2 / 3$ & $6,10,14,21,25,28$ & & $9,13,19$ \\
$4 / 3$ & $1,4,10,14,18,25$ & & 23 \\
$5 / 1$ & $4,9,13,19,22,25$ & $\gamma_{1}$ & 24 \\
$5 / 2$ & $1,8,11,14,18,23$ & $\gamma_{5}$ & 39,55 \\
$5 / 4$ & $7,10,15,21,24,28$ & $\gamma_{15}$ & $33,36,47$ \\
$5 / 5$ & $32,35,39,45,49,55$ & $\gamma_{17}$ & \\
$5 / 6$ & $31,38,41,48,53,56$ & $\gamma_{23}$ & \\
$5 / 7$ & $29,33,36,42,47,52$ & $\gamma_{26}$ & \\
\hline Bits of $K$ & \multicolumn{3}{|c}{$5,20,26,27,30,37,43,44,51$} \\
not affecting $(1)$ & \multicolumn{3}{|c|}{} \\
\hline
\end{tabular}

${ }^{\dagger}$ — These bits appear only once in the computation of $d^{\prime}$ and $d^{\prime \prime}$.

Table 4. Key bits determining the 'middle' bits of 5-round DES

it is sufficient to guess 8 key bits, that along with the 24 bits required for the S-boxes affected by bits 1-28 of the key, are sufficient to determine the values of $d^{\prime}[41-44]$ and $d^{\prime \prime}[41-44]$ up to the value of the four intermediate bits - $\beta_{23}, \beta_{26}$ and $\gamma_{17}, \gamma_{26}$.

Therefore, we can optimize the known plaintext attack to guess 32 bits of the key, along with 4 intermediate encryption bits. The data complexity of the attack in that case is 51 known plaintexts, with time complexity of about $2^{35.5}$ 5 -round DES encryptions. It is possible to guess 4 more key bits in order to determine the value of $\beta_{23}$, thus reducing the data complexity of the attack to 28 known plaintexts while the time complexity is increased to $2^{37.9}$.

\subsection{Using Chosen Plaintexts}

The attacker can choose the plaintexts such that the values of $\beta_{17}, \beta_{23}$ and $\beta_{26}$ are the same for all the plaintexts. Then, the attacker guesses these intermediate bits as part of the key, but can now correlate between the various plaintexts in a much stronger way.

By guessing for each possible guess of the 24 key bits affecting S-boxes $\mathrm{S} 1, \mathrm{~S} 2, \mathrm{~S} 3$, and $\mathrm{S} 4$, and of the three fixed bits $\beta_{17}, \beta_{23}$ and $\beta_{26}$, the attacker tries the 8 possibilities for the unknown $\gamma$ bits. The data complexity of this attack is 8 chosen plaintexts, and the time complexity is about $2^{30} 5$-round DES encryptions. 


\section{Attack on 6-round DES}

Let $d^{\prime}[1-\mathrm{m}]=G_{K}(p)$ and $d^{\prime \prime}[1-\mathrm{m}]=H_{K}^{-1}(c)$. In our attack on 6-round DES, as in attack by Chaum and Evertse, $G_{K}$ consists of the first 3 rounds of DES and $H_{K}$ contains of rounds 4,5 and 6 . Let us consider the intermediary bits $d^{\prime}[5-8]$ and $d^{\prime \prime}[5-8]$.

The analysis of 6-round DES proceeds along the same lines as the analysis of 4-round DES presented in Sect. 4. We present the results of our analysis of 6-round DES in Table 5.

It was observed in [5] that in order to compute $d^{\prime}[5-8]$ and $d^{\prime \prime}[5-8]$, it is sufficient to guess 54 key bits. Thus, if for a key guess the computed values of $d^{\prime}[5-8]$ and $d^{\prime \prime}[5-8]$ disagree, then the key is necessarily wrong, and can be discarded.

Here again, the values of $d^{\prime}[5-8]$ and $d^{\prime \prime}[5-8]$ can be computed by guessing less key bits in exchange for guessing internal bits. We have,

$$
d^{\prime}[5-8]=R_{0}[5-8] \oplus S_{2}\left[E P\left(R_{1}\right)[7-12] \oplus K_{2}[7-12]\right]
$$

and $d^{\prime \prime}[5-8]$ is equal to

$$
\begin{aligned}
d^{\prime \prime}[5-8] & =R_{6}[5-8] \oplus S_{2}\left[E P\left(L_{4}\right)[7-12] \oplus K_{4}[7-12]\right] \\
& \oplus S_{2}\left[E P\left(L_{6}\right)[7-12] \oplus K_{6}[7-12]\right] .
\end{aligned}
$$

We have,

$$
\begin{aligned}
& E P\left(R_{1}\right)[7-12]=\left[\beta_{21} \beta_{29} \beta_{12} \beta_{28} \beta_{17} \beta_{1}\right], \\
& E P\left(L_{4}\right)[7-12]=\left[\gamma_{21} \gamma_{29} \gamma_{12} \gamma_{28} \gamma_{17} \gamma_{1}\right] .
\end{aligned}
$$

If we guess $K_{2}[7-12], K_{4}[7-12]$ and $K_{6}[7-12]$, the unknowns left in the computations of $d^{\prime}[5-8]$ and $d^{\prime \prime}[5-8]$ are $\left[\beta_{21} \beta_{29} \beta_{12} \beta_{28} \beta_{17} \beta_{1}\right]$ and $\left[\gamma_{21} \gamma_{29} \gamma_{12} \gamma_{28} \gamma_{17} \gamma_{1}\right]$.

Consider $\gamma_{1}$. In order to compute this bit we can either guess 2 key bits (of $\left.K_{5}[1-6]\right)$ or guess $\gamma_{1}$ directly. Thus, a different attack algorithm for the meet-inthe-middle attack would be to guess all the 54 key bits suggested by Chaum and Evertse, besides the 2 key bits. For each guess of the 52 key bits, the attacker tries the two possibilities of $\gamma_{1}$. If for both values the equality $d^{\prime}[5-8]=d^{\prime \prime}[5-8]$ is not achieved, then the guess of the 52 bits is necessarily wrong. As for a specific (wrong) guess of the key and of $\gamma_{1}$ the probability of equality is $1 / 16$, the probability that a wrong 52-bit key has at least one $\gamma_{1}$ for which the equality is satisfied is $1-(15 / 16)^{2} \approx 1 / 8$. Hence, the attacker can guess the 52 bits, and by trying the two possibilities of $\gamma_{1}$ reduce the number of remaining candidates to $2^{49}$. Now, using similar techniques as in Sect. 4.1, the number of 6-round DES encryptions to retrieve the full key can be calculated to be $2^{51.8}$. 


\begin{tabular}{|c|c|c|c|}
\hline Round/S-box & Key bits & Bit determined & Bits appearing once $^{\dagger}$ \\
\hline $1 / 1$ & $2,6,12,15,18,25$ & $\beta_{1}$ & \\
\hline $1 / 3$ & $5,9,13,20,24,27$ & $\beta_{12}$ & \\
\hline $1 / 5$ & $32,38,42,48,53,56$ & $\beta_{17}$ & \\
\hline $1 / 6$ & $31,34,41,46,49,52$ & $\beta_{21}$ & \\
\hline $1 / 7$ & $29,35,40,45,50,54$ & $\beta_{28}$ & \\
\hline $1 / 8$ & $30,33,37,43,47,51$ & $\beta_{29}$ & \\
\hline $2 / 2$ & $2,5,8,12,17,23$ & & \\
\hline $4 / 2$ & $6,9,12,16,21,27$ & & \\
\hline $5 / 1$ & $4,9,13,19,22,25$ & $\gamma_{1}$ & 4,19 \\
\hline $5 / 3$ & $3,6,12,16,20,27$ & $\gamma_{12}$ & \\
\hline $5 / 5$ & $32,35,39,45,49,55$ & $\gamma_{17}$ & \\
\hline $5 / 6$ & $31,38,41,48,53,56$ & $\gamma_{21}$ & \\
\hline $5 / 7$ & $29,33,36,42,47,52$ & $\gamma_{28}$ & 36 \\
\hline $5 / 8$ & $30,37,40,44,50,54$ & $\gamma_{29}$ & \\
\hline \multicolumn{4}{|c|}{ There are no key bits of round 6 that appear only once in computing $d^{\prime}$ and $d^{\prime \prime}$. } \\
\hline $\begin{array}{c}\text { Bits of } K \\
\text { not affecting (1) }\end{array}$ & \multicolumn{3}{|c|}{7,28} \\
\hline
\end{tabular}

Table 5. Key bits determining the 'middle' bits of 6-round DES

\section{Conclusions and Open Problems}

In this paper, we have found hitherto unknown weaknesses in block ciphers with up to 6 rounds of DES. We use the meet-in-the-middle technique and improve the time complexities (at the cost of few plaintexts) of similar attacks on DES by Chaum and Evertse [5]. We obtained that the time complexities for key search in the case of 4, 5 and 6-round DES are $2^{20}, 2^{35.5}$ and $2^{51.8}$ using 15,51 and 1 known plaintexts respectively. With 6 chosen ciphertexts and 8 chosen plaintexts the time complexities in the case of 4-round and 5-round attacks are $2^{19.3}$ and $2^{30}$ respectively.

Our research leaves room for alluring open problems. It can be seen from Table 3, Table 4 and Table 5 that Chaum and Evertse have considered bits of the key $K$ that do not appear in the first columns of these tables; we have considered bits of $K$ that appear only once (and sometimes twice) in the first columns. Hence, a natural extension will be to experiment with bits which appear more times in the first columns of these tables. This technique could be tried on DES with higher number of rounds. Another extension of the attacks described in this paper follows a suggestion in [5] by which one may try to change the tables defining the S-boxes. By either of these methods, it could be possible to cryptanalyse DES variants consisting of 8 or more rounds. 


\section{Acknowledgments}

The authors wish to thank the anonymous reviewers of Indocrypt-2007 for their constructive comments on our work.

\section{References}

1. Eli Biham, Alex Biryukov, An Improvement of Davies' Attack on DES, Journal of Cryptology, Vol. 10, No. 3, pp. 195-206, Springer, 1997.

2. Eli Biham, Adi Shamir, Differential Cryptanalysis of DES-like Cryptosystems, Journal of Cryptology, Vol. 4 No. 1, Springer, pp. 3-72, 1991.

3. Eli Biham, Adi Shamir, Differential Cryptanalysis of the Full 16-Round DES, Advances in Cryptology, proceedings of CRYPTO '92, Lecture Notes in Computer Science 740, Springer, 1993.

4. Eli Biham, Adi Shamir, Differential Cryptanalysis of the Data Encryption Standard, Springer, 1993.

5. David Chaum, Jan-Hendrik Evertse, Cryptanalysis of DES with a Reduced Number of Rounds: Sequences of Linear Factors in Block Ciphers, Advances in Cryptology, proceedings of CRYPTO '85, Lecture Notes in Computer Science 218, pp. 192-211, Springer, 1986.

6. CNET News.com, Users take crack at 56-bit crypto. Available on-line at http://news.com.com/2100-1023-278658.html?legacy=cnet, 1997.

7. Nicolas T. Courtois, Gregory V. Bard, Algebraic Cryptanalysis of the Data Encryption Standard. Available on-line at: http://eprint.iacr.org/2006/402.pdf, 2006.

8. Donald W. Davies, Investigation of a Potential Weakness in the DES Algorithm, private communications, 1987.

9. Donald W. Davies, Sean Murphy, Pairs and Triplets of DES S-Boxes, Journal of Cryptology, Vol. 8, No. 1, pp. 1-25, Springer, 1995.

10. Whitfield Diffie, Martin E. Hellman, Exhaustive Cryptanalysis of the NBS Data Encryption Standard, Computer, Vol. 10, No. 6, pp. 74-84, June 1977.

11. Electronic Frontier Foundation, Cracking DES, Secrets of Encryption Research, Wiretap Politics \& Chip Design, O'reilly, 1998.

12. Martin E. Hellman, A Cryptanalytic Time-Memory Tradeoff, IEEE Transactions on Information Theory, Vol. 26, No. 4, pp. 401-406, 1980.

13. Lars R. Knudsen, John E. Mathiassen, A Chosen-Plaintext Linear Attack on $D E S$, proceedings of Fast Software Encryption 7, Lecture Notes in Computer Science 1978, pp. 262-272, Springer, 2001.

14. Sandeep Kumar, Christof Paar, Jan Pelzl, Gerd Pfeiffer, Manfred Schimmler, Breaking Ciphers with COPACOBANA - A Cost-Optimized Parallel Code Breaker, proceedings of CHES 2006, Lecture Notes in Computer Science 4249, pp. 101-118, Springer, 2006.

15. Sebastien Kunz-Jacques, Frederic Muller, New Improvements of Davies-Murphy Cryptanalysis, Advances in Cryptology, proceedings of ASIACRYPT 2005, Lecture Notes in Computer Science 3788, pp. 425-442, Springer, 2005.

16. Mitsuru Matsui, Linear Cryptanalysis Method for DES Cipher, Advances in Cryptology, proceedings of EUROCRYPT '93, Lecture Notes in Computer Science 765, pp. 386-397, Springer, 1994.

17. National Bureau of Standards, Data Encryption Standard, Federal Information Processing Standards Publications No. 46, 1977. 
18. Axel Poschmann, Gregor Leander, Kai Schramm, Christof Paar, New Light-Weight DES Variants Suited for RFID Applications, proceedings of Fast Software Encryption 14, Lecture Notes in Computer Science, Springer (to appear), 2007.

19. Håvard Raddum, Igor Semaev, New Technique for Solving Sparse Equation Systems. Available on-line at: http://eprint.iacr.org/2006/475.pdf, 2006.

20. Adi Shamir, On the Security of DES, Advances in Cryptology, proceedings of CRYPTO '85, Lecture Notes in Computer Science 218, pp. 280-281, Springer, 1986.

21. Takeshi Shimoyama, Toshinobu Kaneko, Quadratic Relation of S-box and Its Application to the Linear Attack of Full Round DES, Advances in Cryptology, proceedings of CRYPTO '98, Lecture Notes in Computer Science 1462, pp. 200-211, Springer, 1998.

22. RSA Data Security, Team of Universities, Companies and Individual Computer Users Linked Over the Internet Crack RSA's 56-Bit DES Challenge. Available on-line at: http://www.rsasecurity.com/news/pr/970619-1.html, 1997.

23. Michael J. Wiener, Efficient DES Key Search, Technical Report TR-244, Carleton University, in Practical Cryptography for Data Internetworks, IEEE Computer Society Press, 1996. First presented at CRYPTO '93 rump session. 